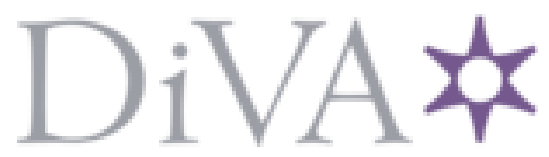

http://www.diva-portal.org

This is the published version of a paper published in European Physical Journal: Applied physics.

Citation for the original published paper (version of record):

Lundström, N., Grafström, A., Aidanpää, J. (2014)

Small shape deviations causes complex dynamics in large electric generators.

European Physical Journal: Applied physics, 66(2): 20903

http://dx.doi.org/10.1051/epjap/2014130447

Access to the published version may require subscription.

N.B. When citing this work, cite the original published paper.

Permanent link to this version:

http://urn.kb.se/resolve?urn=urn:nbn:se:umu:diva-91388 


\title{
Small shape deviations causes complex dynamics in large electric generators
}

\author{
Niklas L.P. Lundström ${ }^{1,2, a}$, Anton Grafström ${ }^{3}$, and Jan-Olov Aidanpää ${ }^{1}$ \\ ${ }^{1}$ Department of Applied Physics and Mechanical Engineering, Luleå University of Technology, SE-97187 Luleå, Sweden \\ 2 Department of Mathematics and Mathematical Statistics, Umeå University, SE-90187 Umeå, Sweden \\ ${ }^{3}$ Department of Forest Resource Management, Swedish University of Agricultural Sciences, SE-90337 Umeå, Sweden
}

Received: 1 October 2013 / Received in final form: 7 March 2014 / Accepted: 28 April 2014

Published online: 5 June 2014 - (C) EDP Sciences 2014

\begin{abstract}
We prove that combinations of small eccentricity, ovality and/or triangularity in the rotor and stator can produce complex whirling motions of an unbalanced rotor in large synchronous generators. It is concluded which structures of shape deviations that are more harmful, in the sense of producing complex whirling motions, than others. For each such structure, we derive simplified equations of motions from which we conclude analytically the relation between shape deviations and mass unbalance that yield non-smooth whirling motions. Finally we discuss validity of our results in the sense of modeling of the unbalanced magnetic pull force.
\end{abstract}

\section{Introduction}

Measurements in large synchronous generators show significant imperfections in the geometry of the air-gap between the generator rotor and stator $[1,2]$. These asymmetries distort the air-gap flux density distribution and therefore produce a force, called unbalanced magnetic pull (UMP), acting between the rotor and the stator. The UMP will affect the dynamics in the generator and may cause damage to the machine. Therefore, to be able to set tolerances for construction and reconstruction of generators it is of importance to investigate the dynamic consequences of geometric irregularities in the air-gap.

In this paper we will use Fourier series to describe the shape of the rotor and stator, and we will focus on the first three frequencies of shape deviations from a perfect circular geometry. These frequencies are referred to as eccentricity, ovality and triangularity. In the case of only eccentricity, a literature survey indicates on intensive studies of methods for calculating UMP, as well as studies on vibrations in machines due to the UMP. To mention a few: [3] and [4] focus on the finite element method (FEM) to find the UMP while [5-8] consider analytical models for the UMP and perform dynamical analysis and simulations. To the authors knowledge, there are only a few papers published considering higher frequencies of shape deviations, see $[4,9-11]$. One reason is probably that eccentricity is easier to study than combinations including higher frequencies since eccentricity forces the rotor to

\footnotetext{
a e-mail: niklas.lundstrom@math.umu.se
}

move in simple motions. In particular, rotor eccentricity yields synchronous whirling, stator eccentricity yields an equilibrium and both imperfections simultaneously yield approximately a synchronous whirling orbit with center at the previously mentioned equilibrium. Due to these facts, the motion created by rotor eccentricity is sometimes referred to as dynamic eccentricity in the literature, while the response produced by stator eccentricity, which is an equilibrium, is called static eccentricity.

However, more complicated whirling motions also exist in large synchronous generators, including both backward whirling, forward whirling and alternating whirling. As already mentioned above, eccentricity alone with an unbalance will not create such a behaviour since these imperfections will force the rotor to a synchronous whirling orbit. However, papers [9-11] show that combinations of shape deviations such as ovality and triangularity can force the rotor to move in orbits including backward whirling, forward whirling and alternating whirling with different whirling frequencies. The above mentioned papers are mainly focused on relatively large deviations of shape on a balanced rotor. In reality there are always other imperfections that forces the rotor to motions, certainly mass unbalance. This motivates the present continuation of the study of dynamic consequences of shape deviations beyond eccentricity, including a mass unbalance. To motivate further we also point out that even if eccentricity is usually dominating the system, the designer may balance the rotor in order to compensate for a synchronous whirling orbit with large amplitude (see [12], p. 4). This may result in a situation where ovality, triangularity or 

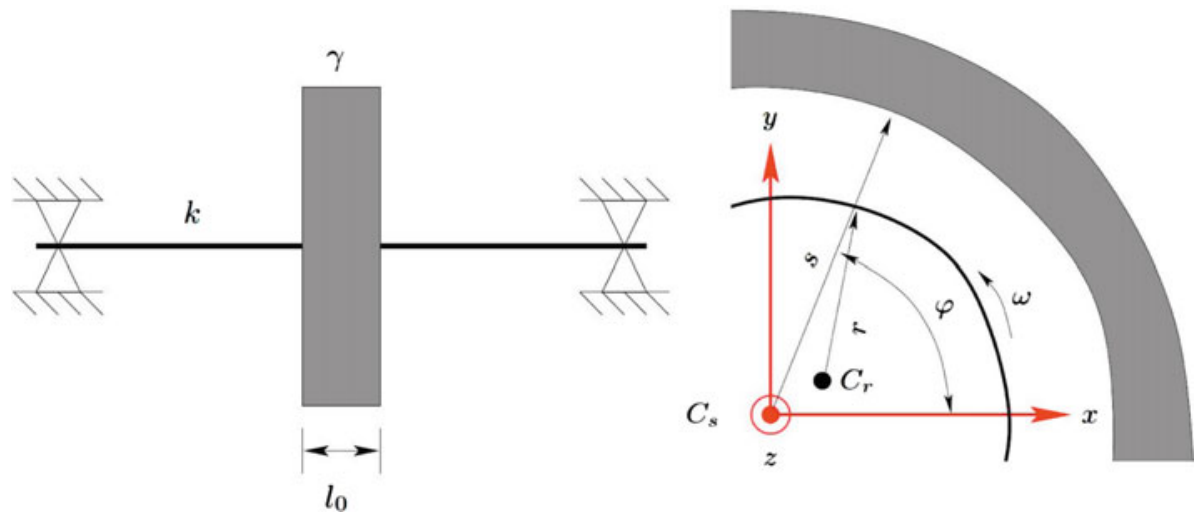

Fig. 1. The Jeffcott rotor (left) and cross-section of the generator (right).

higher frequencies of shape deviations will dominate, since mass unbalance will decrease forces from the rotor eccentricity, see Figure 9 in Section 4.

It is preferable if the generator rotor axis moves in a smooth orbit, such as in synchronous whirling motion, with small amplitude. Non-smooth whirling motions, as shown in Figure $4 \mathrm{f}$ for example, can cause non-uniform wearing and also produce vibrations in the machine. There is also a value of understanding the causes of complex whirling motions in large synchronous generators in general. Using a simple generator model, described in Section 2, we show in Section 3 that complex whirling can be produced by several structures of small shape deviations together with a mass unbalance. We sort out these more harmful structures of shape deviations, see Table 2. Further, for each such structure of shape deviation, we analyze the dynamics as a function of magnitudes on mass unbalance and shape deviation on the rotor and stator. In particular, we derive analytically the relation between shape deviations and mass unbalance that yield a certain whirling motion, see Theorem 1. Finally, we discuss the applicability and generality of our results in Section 4.

\section{Model and method}

The approach is to analyze and numerically simulate the dynamics in a simple generator model to find the key points causing the complicated whirling motions. Then, in Section 4 we consider validity of the model and applicability of our results.

\subsection{Mechanical model}

The generator is treated as a Jeffcott or Laval rotor having a rigid core with length $l_{0}$, mass $\gamma$ and stiffness $k$ of the generator shaft, see Figure 1. The rotor rotates counterclockwise, at a constant angular speed $\omega$. Point $C_{s}$ gives the location of the bearings while point $C_{r}$ is the geometrical center line of the rigid rotor core. The coordinate system has its origin at $C_{s}, r$ is the rotor radius and $s$ is the stator radius.
We assume throughout the paper that the machine is constant in $z$ through the generator length $l_{0}$. Let $r_{0}$ and $s_{0}$ be the average radius of the rotor and the stator respectively. Following [9] the shape irregularities of the rotor radius, $r$, and the stator radius, $s$, will be described by the Fourier series:

$$
\begin{gathered}
r=r_{0}+\sum_{n=1}^{N} \delta_{n}^{r} \cos \left\{n\left(\varphi+\alpha_{n}^{r}\right)\right\}, \\
s=s_{0}+\sum_{m=1}^{M} \delta_{m}^{s} \cos \left\{m\left(\varphi+\alpha_{m}^{s}\right)\right\},
\end{gathered}
$$

where $\delta_{n}^{r} \geq 0, \delta_{m}^{s} \geq 0$ takes small values giving the magnitude of the corresponding frequency of shape deviation and $\alpha_{n}^{r}$ and $\alpha_{m}^{s}$ are the corresponding phase angles. As mentioned in Section 1, we will focus on the three first frequencies of perturbation on the rotor and the stator, that is we let $N=M=3$, and in the following $\delta_{n}^{r}=\delta_{m}^{s}=0$ for all integers $m$ and $n$ if nothing else is mentioned. Moreover, $\delta_{1}^{r}>0$ and $\delta_{1}^{s}>0$ will be referred to as rotor eccentricity and stator eccentricity respectively. Similarly $\delta_{2}^{r}>0$ and $\delta_{2}^{s}>0$ will be referred to as rotor ovality and stator ovality while $\delta_{3}^{r}>0$ and $\delta_{3}^{s}>0$ represent triangularity.

Next, the perturbed air-gap $(g)$ is approximated as:

$$
g=s(\varphi)-r(\varphi)-x \cos \varphi-y \sin \varphi
$$

where $(x, y)$ gives the position of $C_{r}$. Equations (1)-(3) give, after adding the counterclockwise rotation $\omega$,

$$
\begin{aligned}
g= & g_{0}+\sum_{m=1}^{3} \delta_{m}^{s} \cos \left\{m\left(\varphi+\alpha_{m}^{s}\right)\right\} \\
& -\sum_{n=1}^{3} \delta_{n}^{r} \cos \left\{n\left(\varphi+\alpha_{n}^{r}-\omega t\right)\right\}-x \cos \varphi-y \sin \varphi
\end{aligned}
$$

We next introduce the electromagnetic forces acting between the rotor and stator. 


\subsection{Unbalanced magnetic pull}

In electric generators, currents in the rotor and stator create a magnetomotive force giving a magnetic flux (also called B-field) in the air-gap. Summing up the forces in the air-gap may yield a resulting force if the air-gap is non-ideal. This force is called unbalanced magnetic pull (UMP) and is acting roughly in the direction of the narrowest air-gap.

To find a simple analytical expression for the UMP we will assume that the magnetomotive force is constant. This assumption will exclude the periodicity effect induced by the poles. However, this is justified since large synchronous generators usually have a large number of poles (the generator in Tab. 1 has 44 poles). The UMP can now be found as follows. The air-gap variations are given by (4) and the air-gap permeance per unit area is $\mu_{0} / g(x, y, t, \varphi)$, where $\mu_{0}=4 \pi \times 10^{-7}$ is the permeability of free space. The magnetic flux density is given by multiplying the magnetomotive force, now assumed constant $\left(F_{0}\right)$, by the airgap permeance, hence

$$
B(x, y, t, \varphi)=\frac{F_{0} \mu_{0}}{g(x, y, t, \varphi)} .
$$

To find the UMP from the B-field we use the Maxwell stress tensor

$$
\sigma(x, y, t, \varphi)=\frac{B(x, y, t, \varphi)^{2}}{2 \mu_{0}} .
$$

Integrating (6) over the circumference of the generator yields the UMP in the $x$ - and $y$-directions,

$$
\begin{aligned}
& f_{x}=\frac{F_{0}^{2} r_{0}}{2 \mu_{0}} \int_{0}^{2 \pi} \frac{1}{g(x, y, t, \varphi)^{2}} \cos \{\varphi\} \mathrm{d} \varphi \\
& f_{y}=\frac{F_{0}^{2} r_{0}}{2 \mu_{0}} \int_{0}^{2 \pi} \frac{1}{g(x, y, t, \varphi)^{2}} \sin \{\varphi\} \mathrm{d} \varphi .
\end{aligned}
$$

We note that a more detailed derivation of (7) and (8) can be found in [9].

\subsection{Equations of motion and scaling}

The equations of motion for the forced Jeffcott rotor is non-autonomous and nonlinear and consists of the

Table 1. Numerical values.

\begin{tabular}{ccc}
\hline$s_{0}$ & Average stator radius & $2.775 \mathrm{~m}$ \\
$l_{0}$ & Length of the generator & $1.18 \mathrm{~m}$ \\
$g_{0}$ & Average air-gap & $0.0125 \mathrm{~m}$ \\
$\gamma$ & Mass of the rotor & $98165 \mathrm{~kg}$ \\
$k_{m}$ & Magnetic stiffness & $1.4715 \times 10^{8} \mathrm{~N} / \mathrm{m}$ \\
$k$ & Stiffness of the axis & $3.456 \times 10^{8} \mathrm{~N} / \mathrm{m}$ \\
$\omega$ & Rotor rotation speed & $14.2 \mathrm{rad} / \mathrm{s}$ \\
& Number of poles & 44 \\
\hline
\end{tabular}

following two second order differential equations:

$$
\begin{aligned}
& \gamma \ddot{x}+c \dot{x}+k x=h \gamma \omega^{2} \cos \left(\omega t+\alpha_{h}\right)+f_{x}(x, y, t), \\
& \gamma \ddot{y}+c \dot{y}+k y=h \gamma \omega^{2} \sin \left(\omega t+\alpha_{h}\right)+f_{y}(x, y, t) .
\end{aligned}
$$

Here, $\gamma$ is the mass of the rotor, $k$ is the stiffness of the rotor shaft and $c$ being a linear viscous damping. In this paper, $c$ is chosen such that the damping ratio $\zeta$, introduced in (11) below, satisfies $\zeta=0.1$.

In non-dimensional form, system (9) yields

$$
\begin{aligned}
& X^{\prime \prime}+2 \zeta X^{\prime}+X=H \Omega^{2} \cos \left(\Omega \tau+\alpha_{h}\right)+F_{X}(X, Y, \tau), \\
& Y^{\prime \prime}+2 \zeta Y^{\prime}+Y=H \Omega^{2} \sin \left(\Omega \tau+\alpha_{h}\right)+F_{Y}(X, Y, \tau),
\end{aligned}
$$

where prime denotes differentiation with respect to the non-dimensional time $\tau$ and

$$
\begin{aligned}
& X=\frac{x}{g_{0}}, Y=\frac{y}{g_{0}}, H=\frac{h}{g_{0}}, \Delta_{n}^{R}=\frac{\delta_{n}^{r}}{g_{0}}, \\
& \Delta_{m}^{S}=\frac{\delta_{m}^{s}}{g_{0}}, \zeta=\frac{c}{2 \sqrt{k \gamma}}, G=\frac{g(x, y, t, \varphi)}{g_{0}}, \\
& \Omega=\omega \sqrt{\frac{\gamma}{k}}, \tau=t \sqrt{\frac{k}{\gamma}}, K=\frac{k}{k_{m}},
\end{aligned}
$$

are non-dimensional quantities. The air-gap $G$, and the forces $F_{X}$ and $F_{Y}$ yield

$$
\begin{gathered}
F_{X}=\frac{f_{x}}{g_{0} k}=\frac{1}{2 \pi K} \int_{0}^{2 \pi} \frac{\cos \varphi}{G(X, Y, \tau, \varphi)^{2}} \mathrm{~d} \varphi, \\
F_{Y}=\frac{f_{y}}{g_{0} k}=\frac{1}{2 \pi K} \int_{0}^{2 \pi} \frac{\sin \varphi}{G(X, Y, \tau, \varphi)^{2}} \mathrm{~d} \varphi, \\
G=1+\sum_{m=1}^{3} \Delta_{m}^{S} \cos \left\{m\left(\varphi+\alpha_{m}^{s}\right)\right\} \\
-\sum_{n=1}^{3} \Delta_{n}^{R} \cos \left\{n\left(\varphi+\alpha_{n}^{r}-\Omega \tau\right)\right\} \\
-X \cos \varphi-Y \sin \varphi .
\end{gathered}
$$

\subsection{Numerical values and parameters}

The numerical values used in this paper are taken from an $18 \mathrm{MW}$ hydropower generator and are given in Table 1. The magnetic stiffness $k_{m}$ relates to the magnetomotive force $F_{0}$ as follows. For ideal circular generator geometry and $y=0$, the integral in (7) can be solved analytically to yield

$$
f_{x}=\frac{F_{0}^{2} r_{0}}{2 \mu_{0}} \int_{0}^{2 \pi} \frac{\cos \varphi}{\left(g_{0}-x \cos \varphi\right)^{2}} \mathrm{~d} \varphi=k_{m} \frac{x}{\left(1-\frac{x^{2}}{g_{0}^{2}}\right)^{\frac{3}{2}}},
$$

where $k_{m}$ is defined as $k_{m}=\pi F_{0}^{2} r_{0} /\left(\mu_{0} g_{0}^{3}\right)$. The value of $k_{m}$ was found by measuring $f_{x}$ and using (15). The values given in Table 1 are used in all simulations below. 


\subsection{Method for dynamic analysis and notation}

We begin our investigation by first demonstrating complex whirling motions by simple numerical simulation. The function ODE45 in the software MATLAB was used to integrate the dynamical system (10). The size of the basin of attraction to solutions and the question of uniqueness of solutions to system (10) was discussed in [9] and we do not consider those topics in this paper. We refer the reader to [9] for results that strongly indicate that, for small shape deviations, a solution to system (10) is unique and has a large basin of attraction.

Besides numerical simulation, we perform mathematical analysis to strengthen generality of our results. We derive approximating equations of motions that can be solved analytically and thus reveal the behaviour for a large set of parameter values. Finally, we further discuss generality, in particular with respect to the modeling of the UMP, in Section 4.

To be able to present and discuss our results we need to introduce some terminology. Throughout the paper, $E$ will denote the equilibrium point arising when only stator shape deviations are present. The importance of $E$ lies in the fact that adding mass unbalance, and/or rotor shape deviation, will usually force the rotor to an orbit having $E$ as an approximate center. Therefore, we chose to define whirling with respect to $E$ and let

$$
\begin{aligned}
R & =\sqrt{\left(X-X_{E}\right)^{2}+\left(Y-Y_{E}\right)^{2}} \text { and } \\
\Theta & =\arctan \left(\frac{Y-Y_{E}}{X-X_{E}}\right)
\end{aligned}
$$

be polar coordinates, where $\left(X_{E}, Y_{E}\right)$ gives the coordinates of $E$. Next, $\Theta^{\prime}$, where prime denotes differentiation with respect to non-dimensional time $\tau$, is the angular frequency of the motion of the rotor center, with respect to $\left(X_{E}, Y_{E}\right)$. Let $\widehat{\Theta}^{\prime}$ denote the average of $\Theta^{\prime}$ during one revolution of the rotor axis. An orbit will be said to have forward whirling if $\widehat{\Theta}^{\prime}$ is positive and backward whirling if $\widehat{\Theta}^{\prime}$ is negative. An orbit is said to have $n$-whirling if $\widehat{\Theta}^{\prime} / \Omega=n$. Moreover, $n$-whirling is alternating $n$-whirling if $\Theta^{\prime}$ changes sign during one revolution of the rotor. Note that synchronous whirling is 1-whirling. Finally we assume that all shape deviation parameters and mass unbalance is set to zero, that is $\Delta_{m}^{S}=\Delta_{n}^{R}=H=0$ for all integers $m, n$, if nothing else is mentioned. The same is true for the corresponding phase angels, that is we assume $\alpha_{m}^{s}=\alpha_{n}^{r}=\alpha_{h}=0$ if nothing else is mentioned.

\section{Results}

Our main result is that small shape deviations in the rotor and stator can produce complex whirling motions, including backward, forward and alternating whirling, of an unbalanced rotor in large synchronous generators. Considering only one shape deviation frequency (eccentricity,
Table 2. Structures of shape deviation giving complex whirling motions are marked with $X, 1$-whirling with $O$ and at the unmarked positions the effect from the shape deviation is very small due to symmetry. We use the abbreviations $\mathrm{R}$. for rotor and S. for stator.

\begin{tabular}{lccc}
\hline & R. eccentricity & R. ovality & R. triangularity \\
S. eccentricity & $O$ & $X$ & $X$ \\
S. ovality & $O$ & & $X$ \\
S. triangularity & $O$ & $X$ & \\
\hline
\end{tabular}

ovality or triangularity) on the rotor and on the stator, together with a mass unbalance, we sort out which structures of shape deviation that can yield complex whirling motions. We will refer to these cases as harmful structures of shape deviations. For each harmful structure, we analyze the dynamics as a function of magnitudes on mass unbalance and shape deviation on the rotor and stator. We derive analytically the relation between shape deviations and mass unbalance that yield a certain whirling motion, see Theorem 1. In particular, we find which shape deviation and unbalance that yield non-smooth whirling motion.

We summarize the harmful structures of shape deviations in Table 2. In particular, stator eccentricity with rotor ovality, stator eccentricity with rotor triangularity, stator triangularity with rotor ovality and stator ovality with rotor triangularity may produce complex and nonsmooth whirling motions. Rotor eccentricity will always force the machine to more or less smooth 1-whirling, while the UMP will be less harmful due to symmetries in the cases stator ovality with rotor ovality and stator triangularity with rotor triangularity, see [9] and [10].

Given a harmful structure of shape deviation, we now find the magnitudes of mass unbalance and shape deviation producing non-smooth whirling. To do so, we characterize non-smooth whirling as follows. We calculate $\Theta^{\prime}$, with $\Theta$ as in (16), and then we check if $\Theta^{\prime}$ becomes zero during one revolution of the rotor. If so, then the rotor center stops moving and the motion is in that sense nonsmooth. We derive conditions, for each harmful structure, analytically, see Theorem 1 below. Our results are not specific for the generator considered in Table 1. We ensure stability of our results with respect to variations of parameter values by our just mentioned analytical results, and in Section 4 we further discuss generality with respect to the modeling of the UMP.

Figures $2 \mathrm{a}-2 \mathrm{f}$ show the motion of the rotor center when mass unbalance is added to $5 \%$ rotor ovality and $5 \%$ stator eccentricity. When the rotor is balanced the orbit is 2-whirling, around equilibrium $E$, and the machine runs smooth, see Figure 2a. Adding a small mass unbalance to this makes the orbit to separate into two circle-shaped orbits, Figures $2 \mathrm{~b}$ and 2c. Increasing mass unbalance makes the two circle-shaped orbits more different in size and soon the smaller one shrinks to a point where the rotor stops moving, a whirling zero, see Figures $2 \mathrm{~d}-2 \mathrm{f}$. When the mass unbalance becomes larger the response will tend to a synchronous whirling orbit and no more complicated 
N.L.P. Lundström et al.: Small shape deviations causes complex dynamics in large electric generators

(a)

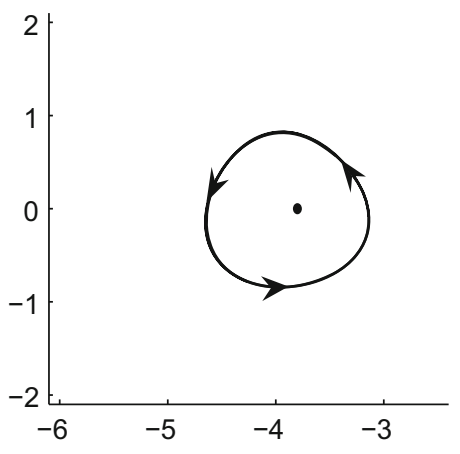

(d)

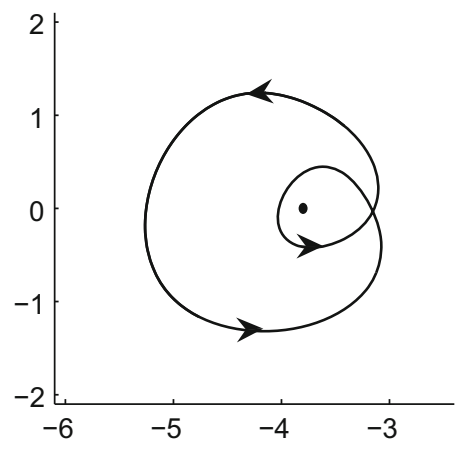

(b)

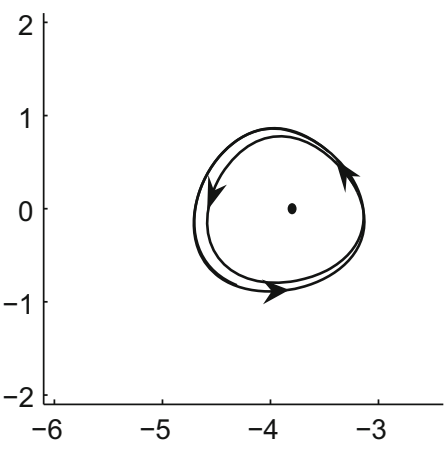

(e)

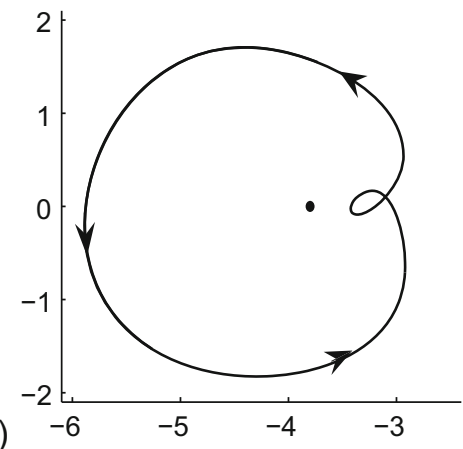

(c)

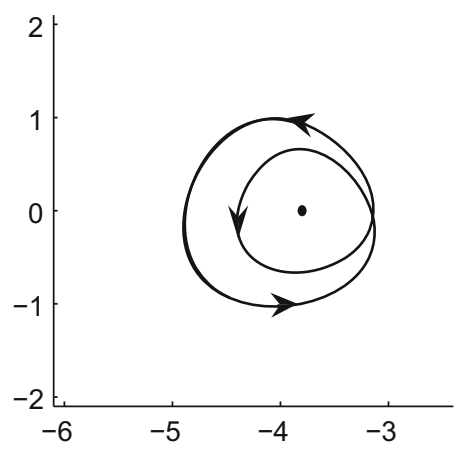

(f)

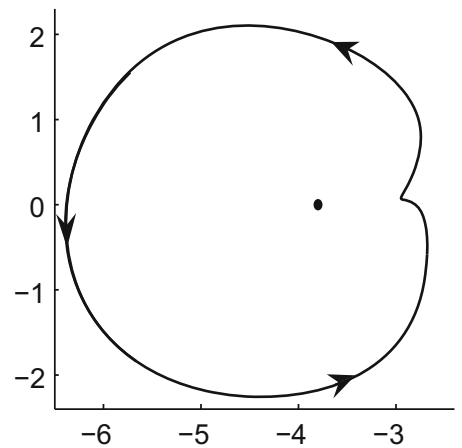

Fig. 2. Motion of the rotor center for $5 \%$ stator eccentricity and $5 \%$ rotor ovality, that is $\Delta_{1}^{S}=\Delta_{2}^{R}=0.05$. The dot represents equilibrium $E$. Mass unbalance is (a) $H=0$, (b) $H=0.005$, (c) $H=0.02$, (d) $H=0.05$, (e) $H=0.1$ and (f) $H=0.14$. Horizontal axis shows $100 \mathrm{X}$ and vertical axis shows $100 Y$.

(a)

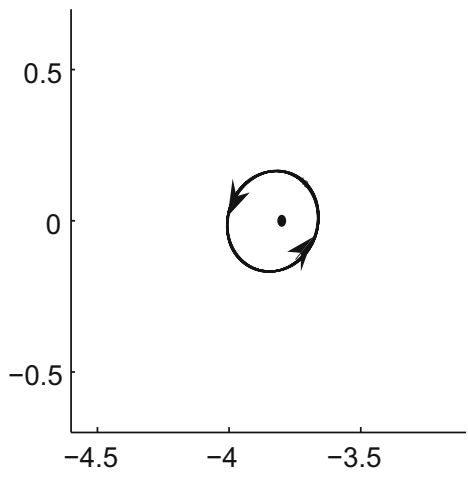

(b)

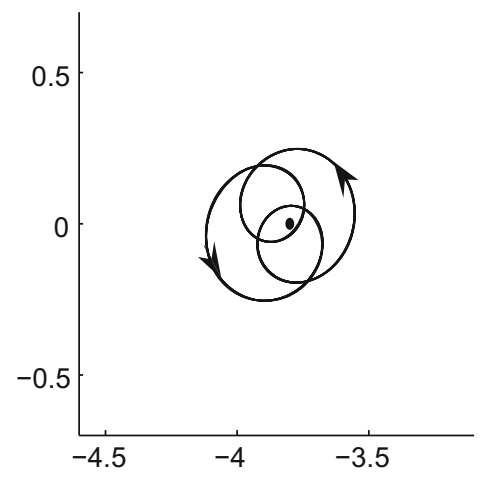

(c)

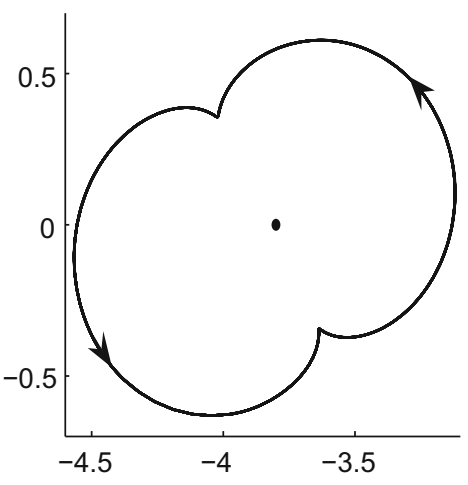

Fig. 3. Motion of the rotor center for $5 \%$ stator eccentricity and $5 \%$ rotor triangularity, that is $\Delta_{1}^{S}=\Delta_{3}^{R}=0.05$. The dot represents equilibrium $E$. Mass unbalance is (a) $H=0$, (b) $H=0.01$ and (c) $H=0.05$. Horizontal axis shows $100 X$ and vertical axis shows $100 Y$.

dynamics will be produced. Figures $3-5$ show that similar motions occur in the other cases summarized in Table 2. The main difference between the harmful structures of shape deviations is the whirling of the UMP and the location of equilibrium $E$. In particular, stator eccentricity with rotor triangularity and stator ovality with rotor triangularity produces 3-whirling, while stator triangularity with rotor ovality yields -2 -whirling. Note that all structures except the last one produces forward whirling. For more on whirling, including higher frequencies of shape deviations but no mass unbalance though, see [10]. When the stator is oval or triangular, equilibrium
$E$ is the origin by symmetry. Only an eccentric stator produces off-centered coordinates of $E$.

Next we try to locate the parameter values giving nonsmooth whirling motions. Figure 6 shows whirling as a function of mass unbalance, rotor ovality and stator triangularity. The curves denotes the existence of a whirling zero, that is $\Theta^{\prime}=0$, as can be seen in Figures $4 \mathrm{~d}-4 \mathrm{f}$. The dotted curves are simulated results while the solid curves are analytical approximations. In Figure 6a shape deviations $\Delta_{2}^{R}=\Delta_{3}^{S}$ and $H$ are varying. Above the curves unbalance is large and forces the motion to 1-whirling, below the curves shape deviations are large and forces the motion 
The European Physical Journal Applied Physics

(a)

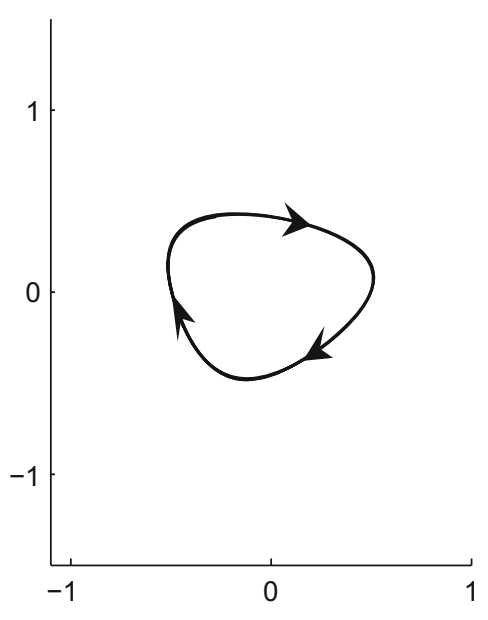

(d)

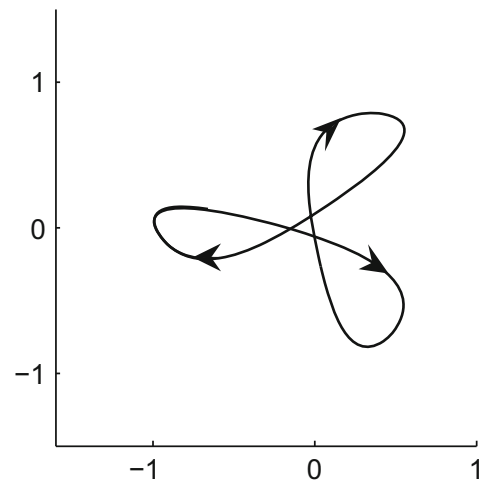

(b)

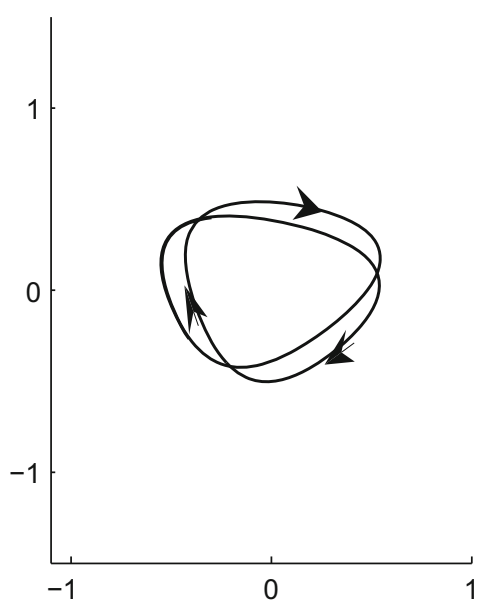

(e)

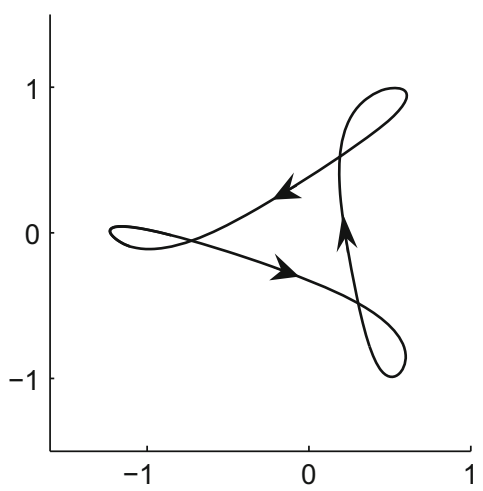

(c)

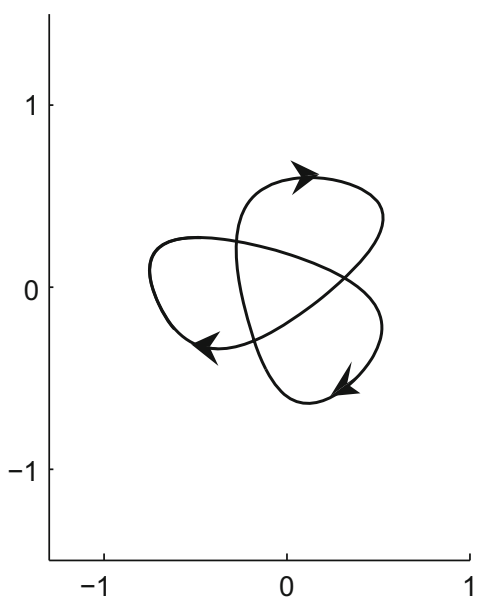

(f)

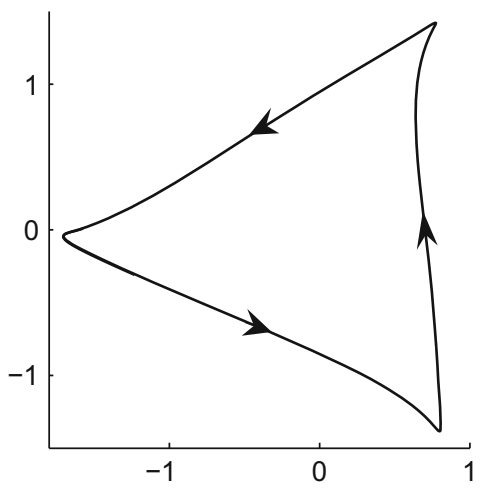

Fig. 4. Motion of the rotor center for $5 \%$ stator triangularity and $5 \%$ rotor ovality, that is $\Delta_{3}^{S}=\Delta_{2}^{R}=0.05$. Equilibrium $E$ is the origin by symmetry. Mass unbalance is (a) $H=0$, (b) $H=0.005$, (c) $H=0.02$, (d) $H=0.04$, (e) $H=0.06$ and (f) $H=0.1$. Horizontal axis shows $100 X$ and vertical axis shows $100 Y$.

(a)

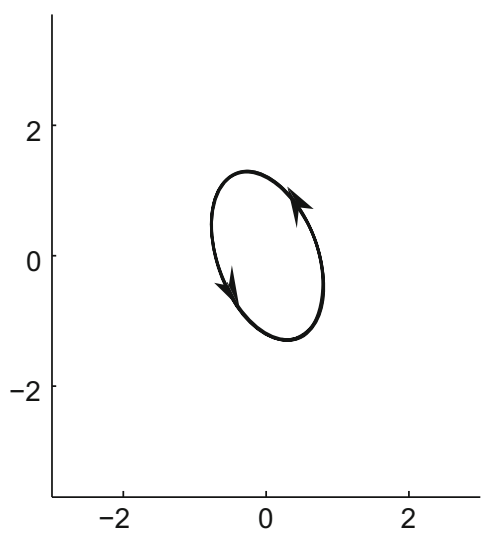

(b)

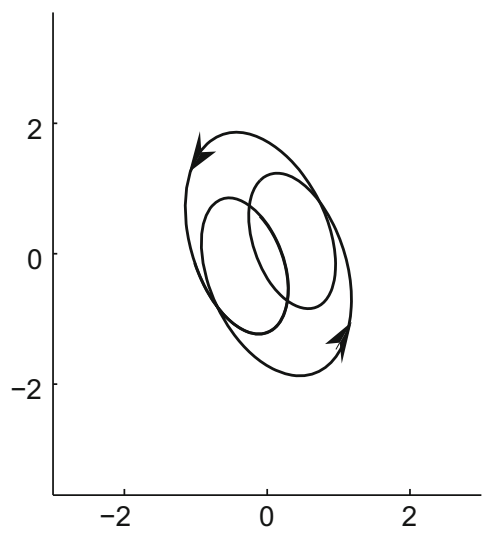

(c)

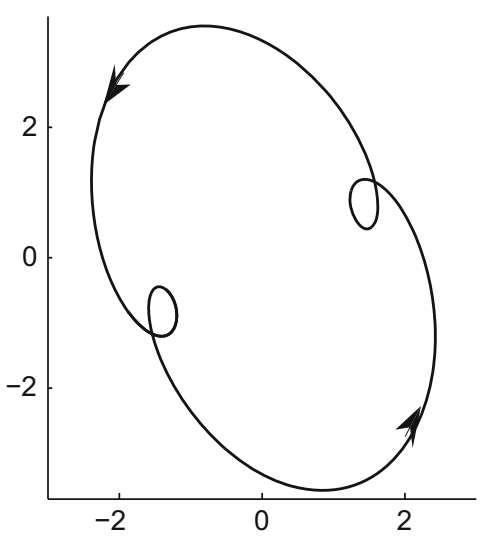

Fig. 5. Motion of the rotor center for $5 \%$ stator ovality and $5 \%$ rotor triangularity, that is $\Delta_{2}^{S}=\Delta_{3}^{R}=0.05$. Equilibrium $E$ is the origin by symmetry. Mass unbalance is (a) $H=0$, (b) $H=0.05$ and (c) $H=0.2$. Horizontal axis shows $100 X$ and vertical axis shows $100 Y$.

to -2 -whirling, between the curves there is alternating non-smooth whirling. In Figure $6 \mathrm{~b}$ unbalance $H=0.05$ is fixed while $\Delta_{2}^{R}$ and $\Delta_{3}^{S}$ are varying. Here, below the curves there is 1 -whirling, above there is -2 -whirling and between there is alternating non-smooth whirling. From Figure 6 and Theorem 1 it is clear that complicated nonsmooth motions will exist for very small shape deviations and mass unbalance, and also for a large set of parameter values, not only those shown in Table 1. Similar results are proven for the other structures of shape deviations, and presented in Theorem 1 below.

Theorem 1 Let $(i, j)=(1,2),(1,3),(3,2)$ or $(2,3)$. Assume that $\Delta_{i}^{S}, \Delta_{j}^{R}, H$ and $\Omega^{2}$ are small and let $\widehat{K}=$ $1-k_{m} / k$. Then the condition for existence of whirling zeroes in solutions to (10) can be well approximated. 

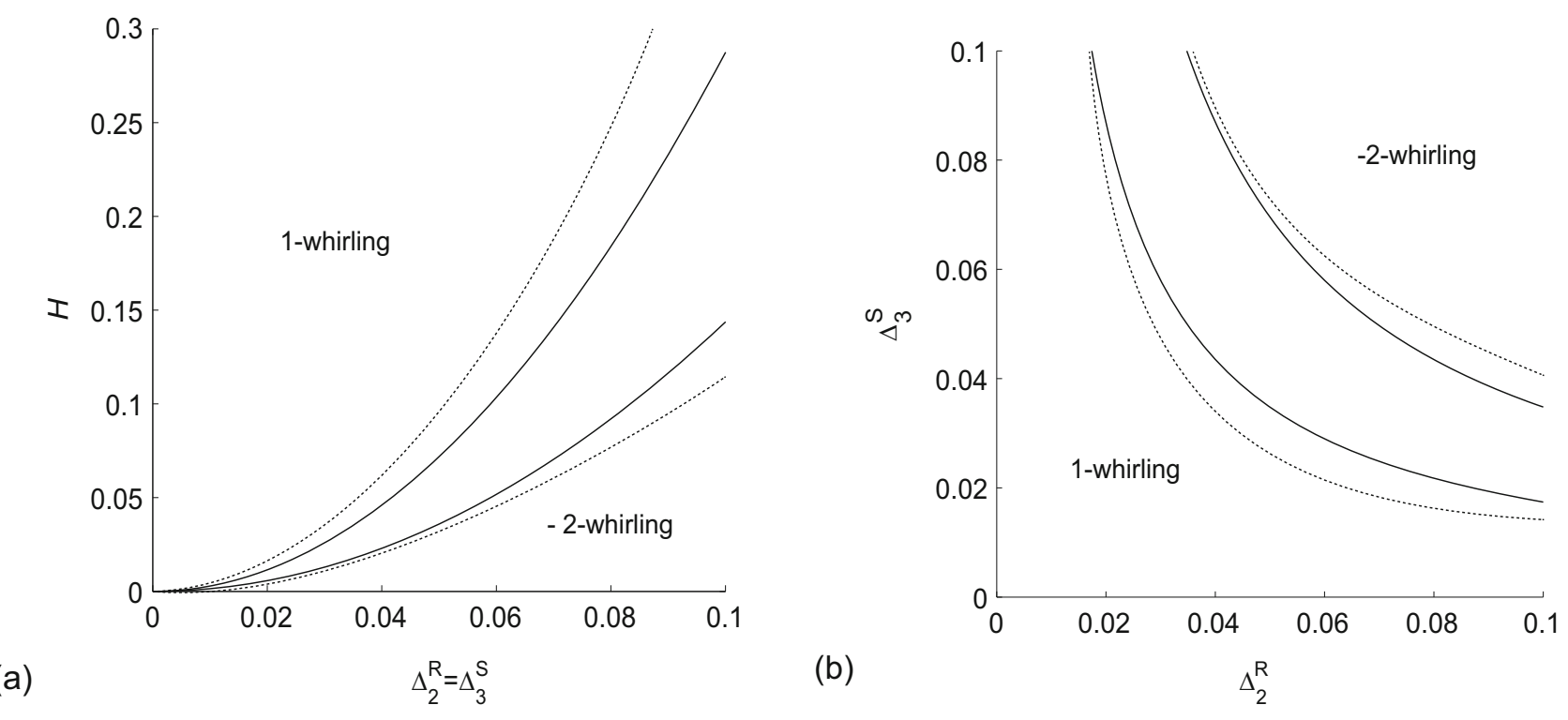

Fig. 6. Existence of whirling zeroes as a function of stator triangularity with rotor ovality and a mass unbalance. The dotted curves are simulated results while the solid curves represent the analytical approximations in Theorem 1 . Between the curves there is alternating non-smooth whirling. (a) Mass unbalance $H$ and shape deviation are varying with $\Delta_{2}^{R}=\Delta_{3}^{S}$. (b) Mass unbalance is fixed to $H=0.05$ and shape deviations are varying.

In particular, there exists at least one whirling zero if

$$
\frac{1}{2} \Gamma_{i, j} \leq \frac{H \Omega^{2}}{3 \Delta_{2}^{R}\left(\Delta_{1}^{S}\right)^{\gamma_{i, j}}} \leq \Gamma_{i, j},
$$

where

$$
\begin{aligned}
\Gamma_{1,2} & =\frac{\widehat{K}^{2}+3 \widehat{K} \Omega^{2}-6 \zeta^{2} \Omega^{2}}{\widehat{K}^{2}(K-1)}, \Gamma_{1,3}=\frac{K}{K-1} \Gamma_{1,2} \\
\Gamma_{3,2} & =\Gamma_{2,3}=\frac{K-1}{K} \Gamma_{1,2}, \gamma_{1,3}=2, \quad \text { and } \\
\gamma_{1,2} & =\gamma_{3,2}=\gamma_{2,3}=1 .
\end{aligned}
$$

The assumptions in Theorem 1 are not hard to satisfy for large synchronous generators since these machines have a low driving frequency $\omega$, making $\Omega^{2}=\omega^{2} \gamma / k$ small. For the parameters in Table $1, \Omega^{2} \approx 0.0573$.

A proof of Theorem 1 is given in Appendix. To prove the theorem we derive approximating linear equations of motion for each structure of shape deviation considered. In particular, see equations (A.3), (A.9), (A.14) and (A.17). These linear equations are then solved explicitly and hence the steady state solutions are given analytically. The simplified equations of motions, derived in the proof of Theorem 1, includes more information than what is stated in the theorem and is therefore of independent interest.

\section{Discussion}

We start by discussing the achieved results and proceed by considering the generality of the modeling of the UMP. Then we discuss the effect of changing the mass unbalance on a generator and finally we consider applications of our results.

We have shown that non-smooth whirling motions, such as whirling zeroes, can be produced by a number of structures of small shape deviations summarized in Table 2. Figure 7 illustrates the speed of the rotor center by showing the location and the angel of the rotor at equidistant time steps. Figures $7 \mathrm{a}-7 \mathrm{c}$ show stator eccentricity with rotor ovality. In Figure 7c the rotor slows down when it enters the smaller circle-shaped orbit, which will collapse into a point when mass unbalance is increased further, recall Figure $2 \mathrm{f}$. In this situation, the whirling zero appears at the least amplitude of the orbit. This is also true for rotor triangularity. Considering instead stator triangularity with rotor ovality, the whirling zeroes appear at the highest amplitude of the orbit, see Figures 7e, $7 \mathrm{f}$ and 4 , therefore this structure of shape deviation is more harmful in the sense of non-smooth whirling. Considering amplitudes, shape deviations including eccentricity will always force to higher amplitudes than higher frequencies of shape deviations due to less symmetry. For more on amplitudes for different structures of shape deviations, see papers $[9,10]$. When comparing the harmful structures, it can be noted that stator eccentricity with rotor triangularity is, in a sense, better than the other cases. This is because the weaker effect of shape deviation which can be seen in Figures 2-5 and 10. This fact is also realized when deriving the analytical approximations; third order terms have to be included in the case stator eccentricity with rotor triangularity, while second order terms are enough in the other cases, see Appendix.

The non-smooth harmful whirling is produced by shape deviations together with mass unbalance since these imperfections forces the rotor by at least two different frequencies. This fact should be clear from the simplified 
(a)

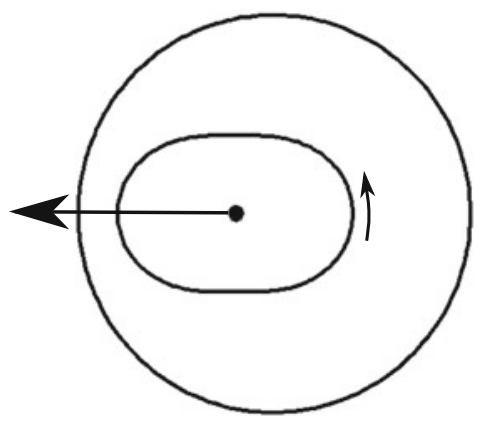

(b)

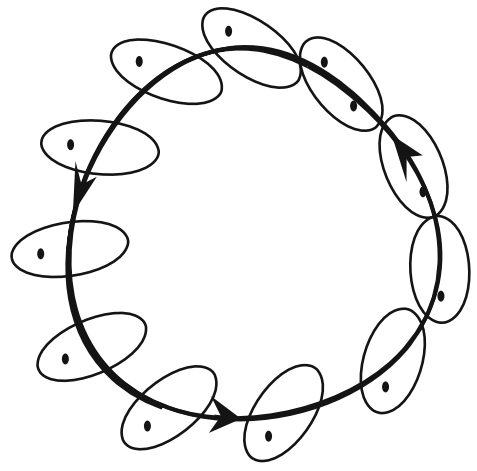

(c)

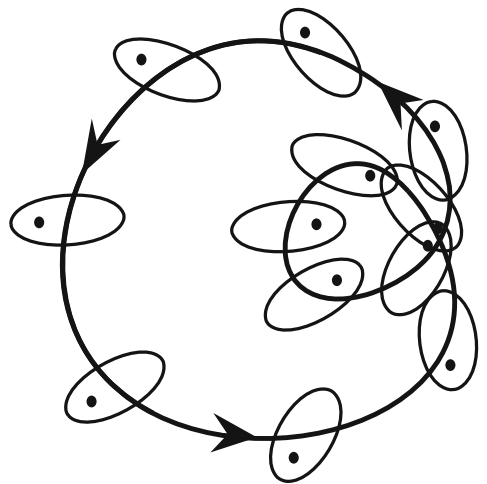

(f)

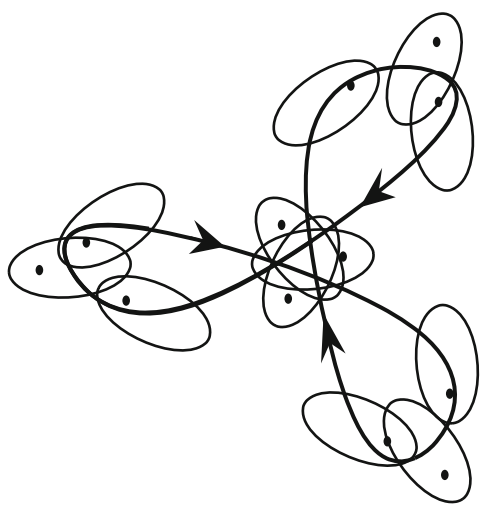

(d)

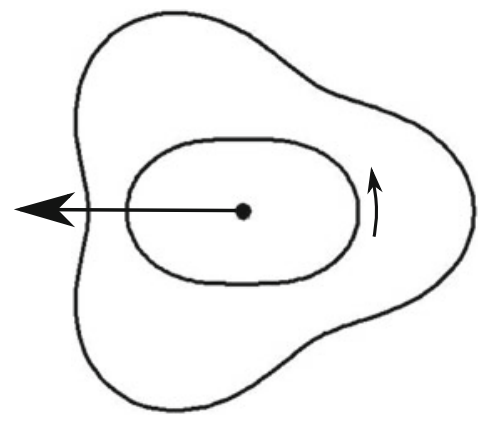

(e)

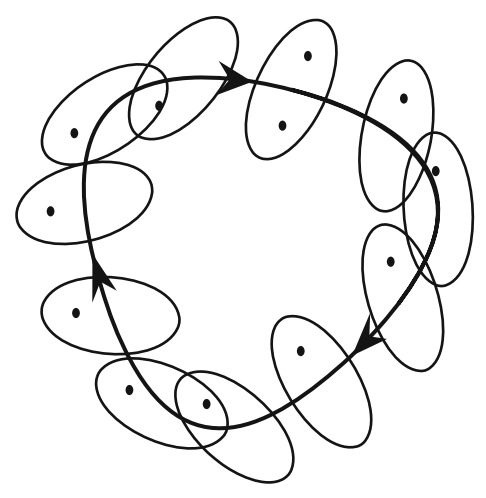

Fig. 7. Subfigures (a) and (d) show the rotor inside the stator with $20 \%$ shape deviation on both the rotor and the stator. The rotor is placed at the origin, and the vector denotes the resulting UMP directed towards the shortest air-gap. Other subfigures show the motion of the rotor center and the angel of the rotor at equidistant time steps for $5 \%$ shape deviation on both the rotor and the stator. The dot represents the heavier (if unbalanced) side of the rotor. In (b) and (e) there is no mass unbalance, in (c) and (f) mass unbalance is $H=0.04$.

equations of motion derived in Appendix, it is simply the different forcing frequencies that produces the whirling zeroes. From this we understand that similar situations will occur when modeling multiphysic situations including forces from bearings, turbines or other shape deviations etc. For example, replacing mass unbalance by rotor eccentricity may yield similar results, since both mass unbalance and rotor eccentricity forces the rotor to 1-whirling. In the same way, an external force with frequency two, minus two, or three times the driving frequency, should give a response similar to rotor ovality with stator eccentricity, rotor ovality with stator triangularity, or rotor triangularity with stator eccentricity or with stator ovality.

\subsection{Modeling of the UMP}

Deriving accurate analytical models for the magnetomotive force, defined in Section 2, and hence for the UMP, is a difficult task. There are also many different constructions of generators that will need different modeling of the UMP, making a general approach including to much details difficult. Some important factors not included in this paper that will significantly affect the UMP are the rotor cage (damper windings), parallel connections of the stator winding and saturation. Variations in the magnetic field will induce circulating currents in the rotor cage and in the parallel connections of the stator winding. These currents will equalize the flux distribution, and therefore reduce the UMP. The direction of the UMP will also change. Currents will be induced in the rotor cage when the magnetic flux changes at a fixed position on the rotor circumference. Hence, in the case of synchronous whirling when the airgap is constant (relative to the rotor) the magnetic flux will also be constant and hence the rotor cage will have no effect. In the case of static eccentricity however, the B-field will vary at each position on the rotor circumference and therefore currents will be induced in the rotor cage which in turn reduce and also change the direction of the UMP. If the generator is equipped with a rotor cage and/or parallel connections of the stator windings, then in most cases of more complicated air-gap variations, such as considered in this paper, currents will be induced in the rotor and/or stator and hence the UMP will be slightly different from what is obtained in Section 2. However, below we will give several arguments saying that most of our results will hold true also in this case, that is, for generators equipped with a rotor cage and/or parallel connections of the stator windings.

Calculating the UMP numerically using FEM seems to be the most popular approach today. For example, see $[3,4]$ and the references therein. Unfortunately, these 

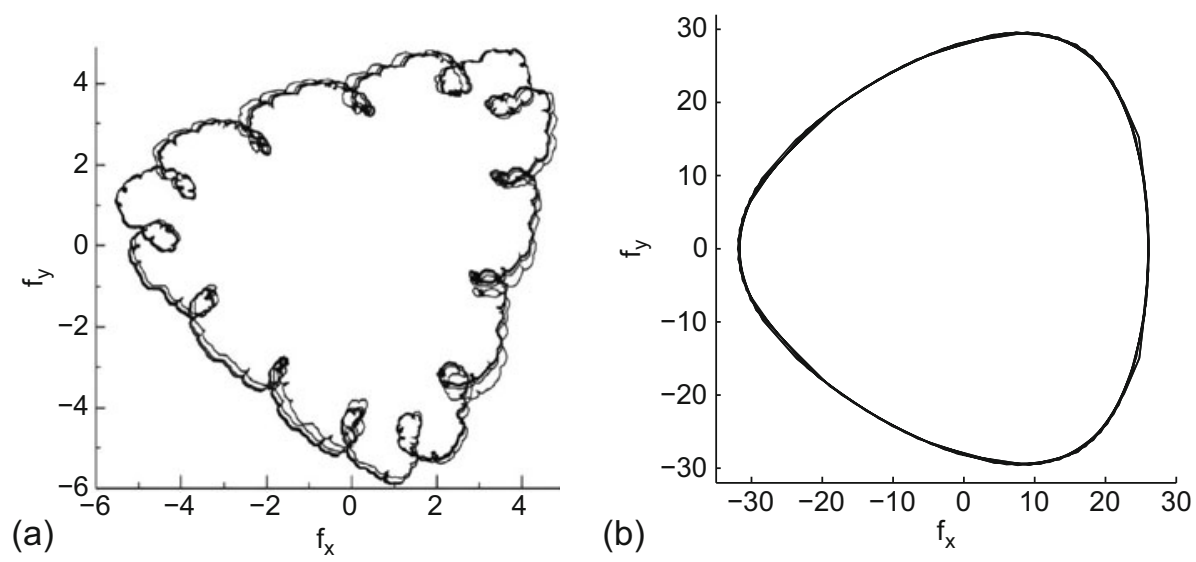

Fig. 8. (a) A FEM simulation of a triangular stator with an oval rotor performed in [4] on a synchronous generator (not the generator in Tab. 1). In particular, this is parts of [4, Fig. 3a]. The effect of the rotor cage is included. (b) A simulation using the present simple analytic model, that is, equations (7) and (8). Rotor ovality and stator triangularity is $10 \%$ in both simulations.

papers often lack explanations of how the electromotive force is produced, which makes those papers difficult to use when trying to find approximating analytical models including more details. However, there are some papers where such models are derived. Using numerical simulations and analytical expressions for currents in the rotor cage and for the currents in the parallel connections in the stator, analytical force models of the UMP are derived in the case of dynamic eccentricity (the rotor center moves in circles around the stator center), in [5] and [13]. Such models have recently been used in [7] to perform dynamic analysis. The models find the UMP as a function of whirling frequency and whirling radius, in the cases with rotor cage and with both rotor cage and parallel connections in the stator. When considering more complicated air-gap geometries, as studied in this paper, a corresponding model has not been found by the authors.

To discuss the generality of our results with respect to the modeling of the UMP we first note that the magnitude of the UMP is not so interesting. The reason is that our main results are existence of complicated asynchronous whirling, for small magnitudes of shape deviations, rather than qualitative parameter intervals. Decreasing or increasing the magnitude of the UMP will not remove existence of the behaviour, but instead moving the parameter interval in which the behaviour occurs. That is, the regions in Figure 6 will slightly move, but not disappear. Therefore, we do not discuss impact on magnitude of the UMP and thus not saturation, which mainly reduces the magnitude of the UMP. Another reason for excluding saturation is that we focus on small shape deviations in this study, and saturation effect becomes strong only when the air-gap is heavily perturbed so that a high magnetic flux density is saturating the material.

Focusing on the impact by the rotor cage on the direction of the UMP when considering static eccentricity, that is, stator eccentricity, the UMP has been shown to change approximately $20^{\circ}$ when adding a rotor cage, see [14]. In the case of whirling motion in a circular orbit around the stator center, the UMP has been shown to change direction up to $20^{\circ}$ due to the rotor cage, see [5]. Considering also parallel paths in the stator, the direction may change up to $30^{\circ}$, see [13] and [15]. In the just mentioned papers, the air-gap variations are very simple. However, since $[5,13,15]$ includes high whirling frequencies, both backward and forward, we believe that the change of the direction of the UMP stays in a similar interval also in our case.

In fact, in [4] a FEM simulation of a triangular stator and an oval rotor is performed. The simulated generator is equipped with a rotor cage and the effect of the currents are included in the simulation. An illustration of this simulation from [4] is given in Figure 8, where we compare it to a simulation using the present modeling of the UMP from Section 2. Ignoring magnitudes and the effect of the poles (note that the generator in [4] is much smaller than the generator described in Tab. 1, and it has only 8 poles giving the higher frequency of the orbit) the differences is mainly a $20^{\circ}$ phase shift. Comparing the result in Figure 8a with Figure $8 \mathrm{~b}$ indicates that the rotor cage gives mainly a change of the direction of the UMP also in more complicated air-gap variations, and the phase shift seems to be rather constant in time. Assuming that this is the case in general, the results in this paper will be nearly identical when including modeling of a rotor cage. It is perhaps more realistic to believe that the phase shift observed in Figure 8 will be time dependent. In such case the time dependence must be periodic, and then the UMP should be approximable by the sum of two or more forces having different frequencies. This would give the possibility to produce even more complex motions. However, since the phase shift seems to be rather small, the additional frequencies will probably not have a large impact.

To proceed the discussion of validity of the UMP modeling, we will now try to explain parts of our results using only the basic fact that the UMP is directed roughly towards the shortest air-gap. This fact is true also when using more detailed methods to find the UMP, as stated 
in $[5,13,15]$, including rotor cage, parallel paths, saturation etc. Hence, the discussion below will strengthen the validity of our results with respect to the UMP modeling.

We start by considering an oval rotor in an eccentric stator as shown in Figure 7a. The resulting UMP, directed towards the shortest air-gap ( $\pi$ radians), is marked by the arrow. When the rotor starts to rotate counterclockwise, the shortest air-gap will do so as well, and hence also the UMP. Due to the rotor ovality, the UMP must rotate with mean frequency twice the driving frequency of the rotor. Therefore, this shape deviation will produce 2-whirling, as seen in Figure 7b. The whirling will be forward, not backward, because the shortest air-gap will decrease when the rotor starts to rotate from the position in Figure $7 \mathrm{a}$, and then the UMP will also decrease. Thus, when the rotor starts to rotate counterclockwise, then the rotor center moves down and to the right, producing forward whirling. When unbalance is added, the orbit separates into a twoperiodic motion because the centrifugal force will help the UMP one period, and fight against the UMP during the next period, see Figures $7 \mathrm{c}$ and $2 \mathrm{~b}$. At a certain relation between shape deviation and mass unbalance, the forces cancels out and the smaller circle-shaped orbit shrinks to a point where the rotor center stops moving, recall Figure 2f.

Next, we consider rotor ovality with stator triangularity. The resulting UMP is directed $\pi$ radians when the rotor is placed at the origin, see Figure $7 \mathrm{~d}$. By symmetry, when the rotor has rotated counterclockwise $\pi / 3$ radians, the resulting force has rotated clockwise and is now directed $\pi / 3$ radians. Similarly, when the rotor has angle $2 \pi / 3$, the force has angle $5 \pi / 3$. After one half revolution of the rotor, the initial case in Figure $7 \mathrm{~d}$ is recovered. Hence, when the rotor rotates counterclockwise, the force will rotate clockwise with twice the angular frequency and therefore produce backward whirling, in particular -2-whirling, see Figure 7e. To understand the motion in Figure $7 \mathrm{f}$ we first consider a case when mass unbalance is large and forces the motion to 1-whirling, recall Figure 4f. The triangularity of the stator forces the motion towards a triangular shaped orbit. Decreasing mass unbalance will produce a situation where the UMP, as described in Figure 7e, starts to affect the motion more and more. In the middle of the straight parts of the orbit in Figures 4e and $4 \mathrm{f}$ the shortest air-gap, and hence also the UMP, are opposite directed to the mass unbalance force. Therefore when mass unbalance decreases, the straight parts bends more and more. In the corners both forces has the same direction for a moment, and the UMP rotates clockwise while the mass unbalance force rotates counterclockwise. Therefore, when the mass unbalance decreases the UMP starts to produce backward whirling near the corners. Note also that the shortest air-gap, and hence also the strongest UMP, will be produced at the corners of the orbit, and the mass unbalance helps the UMP to force the rotor away from the origin at the corners, producing the slow motions at large amplitudes and so the non-smooth motion of the machine. The cases of shape deviation including rotor triangularity can be explained in a similar discussion.

\subsection{Changing the mass unbalance}

Given a generator operating at approximately synchronous whirling, the amplitude can be decreased by adding a mass unbalance to the rotor in the direction of the largest air-gap. If the generator has some degree of harmful shape deviation, beyond eccentricity as those investigated in this paper, a negative consequence will be that now these higher frequencies of shape deviations may take over and determine the dynamics. The consequences can be complex asynchronous whirling motions, as seen in Figures $2-5$. In particular, designers may use this to remove synchronous whirling with large amplitudes (see [12], p. 4). Therefore, even if a machine has a large rotor eccentricity, one should not ignore higher deviations of shape when trying to explain the dynamics since mass unbalance may be adjusted to cancel the effect of rotor eccentricity. Opposite, given a generator operating at some unwanted asynchronous whirling, adding a mass unbalance may, if not producing a dangerously high amplitude, force the motion to smooth synchronous whirling.

Figure 9 shows the motion of the rotor center for different mass unbalance, for a generator with a combination

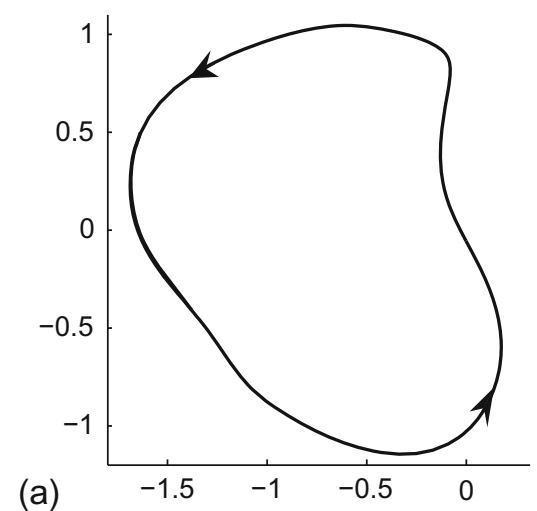

(b)
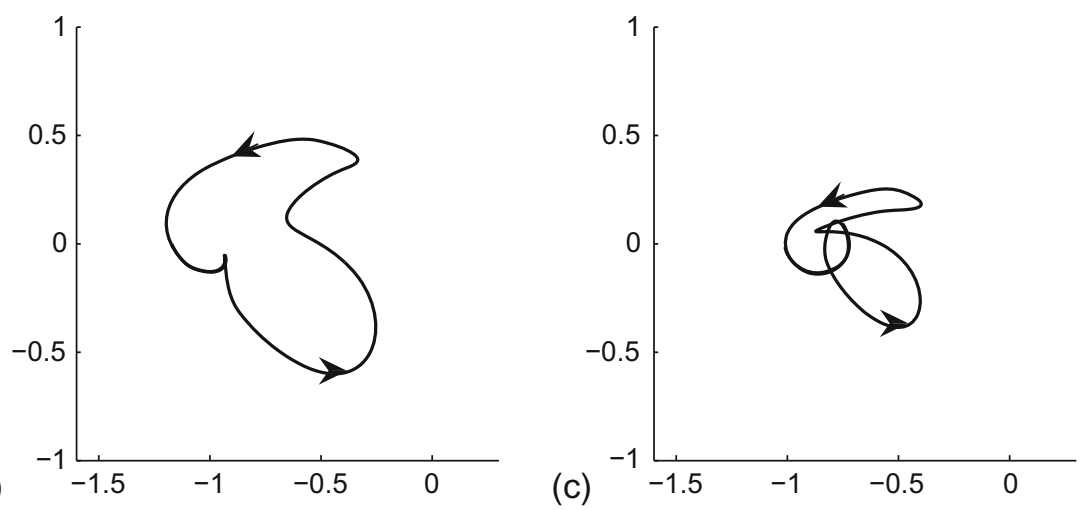

Fig. 9. Mass unbalance added to reduce amplitude. Therefore $\alpha_{h}=\pi$ and the magnitudes are set to (a) $H=0$, (b) $H=0.05$ and (c) $H=0.07$. Rotor and stator eccentricity is $1 \%$, ovality and triangularity are $2 \%$ on both the rotor and the stator. Horizontal axis shows $100 X$ and vertical axis shows $100 Y$. 
of eccentricity, ovality and triangularity on both the rotor and the stator. In Figure 9a there is no mass unbalance and rotor eccentricity produces approximately synchronous whirling. In Figures $9 \mathrm{~b}$ and $9 \mathrm{c}$ a mass unbalance is added to reduce the impact of the rotor eccentricity. The result is complex whirling produced by higher frequencies of shape deviation.

The non-smooth harmful whirling is produced by shape deviations together with mass unbalance since these imperfections forces the rotor by at least two different frequencies. This fact should be clear from the simplified equations of motion derived in Appendix, it is simply the different forcing frequencies that produces the whirling zeroes.

\subsection{Application of our results}

Given a generator operating with asynchronous complex whirling, the reason may be that the machine has some of the harmful structures of shape deviations marked with $X$ in Table 2 . The motion may be explained by measuring the shapes of the rotor and stator and then comparing to the results given in this paper. Using the presented model it is also easy to simulate any measured rotor and stator shape. However, it is probably rather difficult to relate a measured motion to a simulated one, except if only a few frequencies of shape deviations are dominating all imperfections in the machine. The situation in Figure 9 indicates this just mentioned difficulty by the complex motion and also the sensitivity to changes in mass unbalance. In addition, other forces, for example from bearings and turbines will also make the situation more complex.

The results found in this paper can be used during restore work on old generators and when constructing new machines. Then the harmful structures of shape deviations in Table 2 can be removed from the machine in order to prevent harmful non-smooth and complex whirling motions. Moreover, for each harmful structure of shape deviation, we derive approximating linear equations of motion in Appendix. These simplified equations, or the simplified expressions for the UMP, can be used in more general situations such as multiphysic modeling including effects from shape deviations, turbines and bearings etc.

\section{Conclusions}

We have shown that small shape deviations in the rotor and stator can produce complex non-smooth whirling motions, including backward, forward and alternating whirling, of an unbalanced rotor in large synchronous generators. We have sorted out which structures of shape deviations that are more harmful than others, see Table 2. For each harmful structure, we derived analytically the relation between shape deviations and mass unbalance that yield a certain whirling motion, see Theorem 1 . In particular, we determine which shape deviation and unbalance that yield non-smooth whirling motion.
The results presented in this paper give basic understanding of the causes of complex whirling motions in large synchronous generators. During restore work and when constructing new machines, our results can be used to remove harmful shape deviations from the machine in order to prevent non-smooth whirling motions.

\section{References}

1. P. Talas, P. Toom, IEEE Trans. Power Appar. Syst. 9, 3098 (1983)

2. D.V. Kulikov, Yu.A. Anikin, S.V. Dvoinishnikov, V.G. Meledin, Power Technol. Eng. 44, 416 (2011)

3. A. Arkkio, M. Antila, K. Pokki, A. Simon, E. Lantto, IEEE Proc. Elect. Power. Appl. 147, 353 (2000)

4. U. Lundin, A. Wolfbrandt, IEEE Trans. Magn. 45, 2976 (2009)

5. A. Burakov, A. Arkkio, Electr. Eng. 89, 127 (2006)

6. G. Traxler-Samek, T. Lugand, M. Uemori, IEEE Trans. Ind. Electron. 59, 2258 (2012)

7. Y. Calleecharan, J.-O. Aidanpää, IET Sci. Meas. Technol. 5, 231 (2011)

8. M. Karlsson, Doctoral Thesis 2008:27, ISSN: 1402-1544, ISRN: LTU-DT-08/27-SE

9. N.L.P. Lundström, J.-O. Aidanpää, J. Sound Vib. 301, 207 (2007)

10. N.L.P. Lundström, J.-O. Aidanpää, Int. J. Non-Linear Mech. 43, 933 (2008)

11. N.L.P. Lundström, J.-O. Aidanpää, Acta Mech. Sin. 27, $18(2011)$

12. R.K. Gustavsson, J.-O. Aidanpää, Using Strain Gauges to Measure Load on Hydro Generator Guide Bearings (Hydro Review Worldwide, 2003)

13. A. Burakov, A. Arkkio, IEE Proc. Elect. Power Appl. 153, $592(2006)$

14. L. Lundström, R. Gustavsson, J.-O. Aidanpää, N. Dahlbäck, M. Leijon, IET Electr, Power Appl. 1, 1 (2007)

15. A. Burakov, A. Arkkio, IEEE Trans. Magn. 43, 4083 (2007)

\section{Appendix}

\section{A. Proof of Theorem 1}

To prove Theorem 1 we find, for the four structures of shape deviations, linear equations of motions which approximates (10) good enough to yield the desired information. These equations of motions are then solved analytically, and given the solutions we find the inequalities in Theorem 1 as the condition for existence of a whirling zero. Finally we give some simulations to convince the reader that solutions to the approximating systems agrees well with solutions to the original equations of motion (10).

To simplify the expressions for the UMP, consider the Maclaurin series

$$
\frac{1}{(1-\epsilon)^{2}}=1+2 \epsilon+3 \epsilon^{2}+\cdots+(q+1) \epsilon^{q}+\ldots
$$


The European Physical Journal Applied Physics

with

$$
\begin{aligned}
\epsilon= & -\Delta_{m}^{S} \cos \{m \varphi\}+\Delta_{n}^{R} \cos \{n(\varphi-\Omega \tau)\} \\
& +X \cos \varphi+Y \sin \varphi
\end{aligned}
$$

Using (12) and (A.1), $F_{X}$ (and similarly $F_{Y}$ ) can be expressed as:

$$
\begin{gathered}
F_{X}=\frac{1}{2 \pi K} \int_{0}^{2 \pi} \quad\left(1+2 \epsilon+3 \epsilon^{2}+\cdots+(q+1) \epsilon^{q}+\ldots\right) \\
\quad \times \cos \{\varphi\} \mathrm{d} \varphi=F_{X}^{1}+F_{X}^{2}+\cdots \\
+F_{X}^{q}+\ldots
\end{gathered}
$$

We proceed by considering each structure of shape deviation separately.

\section{A.1 Stator eccentricity with rotor ovality}

In this case we obtain $F_{X}^{1}=\left(X-\Delta_{1}^{S}\right) / K$ and $F_{Y}^{1}=Y / K$. Hence, first order terms yield no information about the rotor ovality and therefore we need to include also the second order terms, which simplifies to

$$
\begin{aligned}
& F_{X}^{2}=\frac{3 \Delta_{2}^{R}}{2 K}\left(Y \sin \{2 \Omega \tau\}+\left(X-\Delta_{1}^{S}\right) \cos \{2 \Omega \tau\}\right), \\
& F_{Y}^{2}=\frac{3 \Delta_{2}^{R}}{2 K}\left(\left(X-\Delta_{1}^{S}\right) \sin \{2 \Omega \tau\}-Y \cos \{2 \Omega \tau\}\right) .
\end{aligned}
$$

We first consider the case of a balanced perfectly shaped rotor, that is $H=\Delta_{2}^{R}=0$. In such case the forces are independent of time and hence the solution must be, if stable, an equilibrium point. As before we denote this equilibrium by $E$. Using (10), $F_{X}^{1}, F_{Y}^{1}, F_{X}^{2}$ and $F_{Y}^{2}$ above, we approximate the coordinates of $E$ as the solution to

$$
X=F_{X}^{1}+F_{X}^{2}=\frac{X-\Delta_{1}^{S}}{K}, \quad Y=F_{Y}^{1}+F_{Y}^{2}=\frac{Y}{K},
$$

which is $X_{E}=-\Delta_{1}^{S} /(K-1)$ and $Y_{E}=0$. If $\Delta_{1}^{S}, \Delta_{2}^{R}$ and $H \Omega^{2}$ are small, then adding mass unbalance and/or rotor ovality to this makes the rotor center to move in an orbit around equilibrium $E$. To find approximating equations of motions, we now plug in $(X, Y)=\left(X_{E}, Y_{E}\right)$ into $F_{X}^{2}$ and $F_{Y}^{2}$. Then, using (10), (A.2), $F_{X}^{1}, F_{Y}^{1}, F_{X}^{2}$ and $F_{Y}^{2}$, the simplified linear system yields, with $\widehat{K}=1-K^{-1}$,

$$
\begin{aligned}
X^{\prime \prime}+2 \zeta X^{\prime}+\widehat{K} X= & H \Omega^{2} \cos \left\{\Omega \tau+\alpha_{h}\right\} \\
& -\frac{\Delta_{1}^{S}}{K}-\frac{3 \Delta_{2}^{R} \Delta_{1}^{S}}{2(K-1)} \cos \{2 \Omega \tau\} \\
Y^{\prime \prime}+2 \zeta Y^{\prime}+\widehat{K} Y= & H \Omega^{2} \sin \left\{\Omega \tau+\alpha_{h}\right\} \\
& -\frac{3 \Delta_{2}^{R} \Delta_{1}^{S}}{2(K-1)} \sin \{2 \Omega \tau\} .
\end{aligned}
$$

From (A.3) we realize that the solution orbit for the approximating linear equations of motion is simply the sum of two frequencies; 1 -whirling from the mass unbalance and $2-$ whirling from the shape deviations. Equation (A.3) has a steady state solution of the form

$$
\begin{aligned}
X(t)= & X_{E}+R_{H} \cos \left\{\Omega \tau+\alpha_{h}-v_{H}\right\} \\
& +R_{\Delta}^{X} \cos \left\{2 \Omega \tau-v_{\Delta}\right\}, \\
Y(t)= & Y_{E}+R_{H} \sin \left\{\Omega \tau+\alpha_{h}-v_{H}\right\} \\
& +R_{\Delta}^{Y} \sin \left\{2 \Omega \tau-v_{\Delta}\right\},
\end{aligned}
$$

where $X_{E}, Y_{E}, R_{H}, v_{H}, R_{\Delta}^{X}, R_{\Delta}^{Y}$ and $v_{\Delta}$ yield

$$
\begin{aligned}
X_{E} & =-\frac{\Delta_{1}^{S}}{K-1}, \quad Y_{E}=0 \\
R_{H} & =\frac{H \Omega^{2}}{\sqrt{\left(\widehat{K}-\Omega^{2}\right)^{2}+4 \zeta^{2} \Omega^{2}}}, \\
v_{H} & =\arctan \left\{\frac{2 \zeta \Omega}{\widehat{K}-\Omega^{2}}\right\}, \\
R_{\Delta} & =R_{\Delta}^{X}=R_{\Delta}^{Y}=\frac{2(K-1) \sqrt{\left(\widehat{K}-4 \Delta_{2}^{2}\right)^{2}+16 \Omega^{2} \zeta^{2}}}{2} \\
v_{\Delta} & =\arctan \left\{\frac{4 \zeta \Omega}{\widehat{K}-4 \Omega^{2}}\right\}+\pi .
\end{aligned}
$$

To find a condition for whirling zeroes we ask for the existence of solutions to the equation $Y^{\prime} \widehat{X}-\widehat{X}^{\prime} Y=0$, where $\widehat{X}=X-X_{E}$. Using (A.4) we obtain

$$
\frac{R_{H}^{2}+2 R_{\Delta}^{2}}{3 R_{H} R_{\Delta}}=-\cos \left\{\Omega \tau-\alpha_{h}+v_{H}-v_{\Delta}\right\} .
$$

Note that (A.6) has either no solution, one solutions (if left hand side is 1) or two solutions symmetrically located in the $X Y$-plane, as expected from the simulations in Figure 2. The condition for existence is

$$
R_{H}^{2}+2 R_{\Delta}^{2} \leq 3 R_{H} R_{\Delta}
$$

Now divide (A.7) by $\left(\Delta_{2}^{R} \Delta_{1}^{S}\right)^{2}$, put $z=H \Omega^{2} /\left(\Delta_{2}^{R} \Delta_{1}^{S}\right)$ and write the condition as $a z^{2}-b z+c \leq 0$ where $a, b$ and $c$ are positive expressions following from (A.5) which are independent of the parameters $H, \Delta_{2}^{R}$ and $\Delta_{1}^{S}$. We obtain:

$$
\frac{b}{2 a}-\sqrt{\frac{b^{2}}{4 a^{2}}-\frac{c}{a}} \leq z \leq \frac{b}{2 a}+\sqrt{\frac{b^{2}}{4 a^{2}}-\frac{c}{a}},
$$

and after some calculations we find that

$$
\frac{b}{2 a} \pm \sqrt{\frac{b^{2}}{4 a^{2}}-\frac{c}{a}}=\frac{3(3 \pm 1)}{4(K-1)} \sqrt{\frac{\left(\widehat{K}-\Omega^{2}\right)^{2}+4 \zeta^{2} \Omega^{2}}{\left(\widehat{K}-4 \Omega^{2}\right)^{2}+16 \zeta^{2} \Omega^{2}}} .
$$

To simplify algebraic expressions we perform one last simplification, using the fact that $\Omega^{2}$ is small. In particular, using the Maclaurin series

$$
\sqrt{\frac{(\widehat{K}-\epsilon)^{2}+4 \zeta^{2} \epsilon}{(\widehat{K}-4 \epsilon)^{2}+16 \zeta^{2} \epsilon}}=1+\frac{3\left(\widehat{K}-2 \zeta^{2}\right)}{\widehat{K}^{2}} \epsilon+O\left(\epsilon^{2}\right)
$$

with $\epsilon=\Omega^{2}$, ignoring $O\left(\Omega^{4}\right)$ terms and higher and plugging in the result into (A.8) gives the desired inequality. 
We summarize the assumptions needed for the approximation to be well satisfied. In particular, it is enough to assume that $\Delta_{1}^{S}, \Delta_{2}^{R}, H$ and $\Omega^{2}$ are small.

\section{A.2 Stator eccentricity with rotor triangularity}

Again we obtain $F_{X}^{1}=\left(X-\Delta_{1}^{S}\right) / K$ and $F_{Y}^{1}=Y / K$. In this case the second order terms vanish, that is $F_{X}^{2}=F_{Y}^{2}=0$. Hence, in order to obtain enough information we include also third order terms in (A.2), which yield

$$
\begin{aligned}
F_{X}^{3}= & -\frac{3}{2 K}\left[-2 \Delta_{3}^{R} Y\left(X-\Delta_{S}\right) \sin \{3 \Omega \tau\}\right. \\
& +\Delta_{3}^{R}\left(Y^{2}-\left(X-\Delta_{1}^{S}\right)^{2}\right) \cos \{3 \Omega \tau\} \\
& \left.-\left(X-\Delta_{1}^{S}\right)\left(\left(X-\Delta_{1}^{S}\right)^{2}+Y^{2}+2\left(\Delta_{3}^{R}\right)^{2}\right)\right], \\
F_{Y}^{3}= & \frac{3}{2 K}\left[-2 \Delta_{3}^{R} Y\left(X-\Delta_{S}\right) \cos \{3 \Omega \tau\}\right. \\
& -\Delta_{3}^{R}\left(Y^{2}-\left(X-\Delta_{1}^{S}\right)^{2}\right) \sin \{3 \Omega \tau\} \\
& \left.+\left(X-\Delta_{1}^{S}\right)\left(\left(X-\Delta_{1}^{S}\right)^{2}+Y^{2}+2\left(\Delta_{3}^{R}\right)^{2}\right)\right] .
\end{aligned}
$$

Again we first consider the case $H=\Delta_{3}^{R}=0$ when the solution is an equilibrium point $E$. Using (10) and $F_{X}^{k}, F_{Y}^{k}$ $(k=1,2,3)$ above to find the equilibrium yields complicated algebraic expressions, and therefore we approximate by ignoring third order terms. This results in the same expressions as in the former case, that is $X_{E}=-\Delta_{1}^{S} /(K-1)$ and $Y_{E}=0$. Since $\Delta_{1}^{S}, \Delta_{2}^{R}$ and $H \Omega^{2}$ are assumed small, adding mass unbalance and/or rotor triangularity to this makes the rotor center to move in an orbit around equilibrium $E$. Put $(X, Y)=\left(X_{E}, Y_{E}\right)$ into $F_{X}^{3}, F_{Y}^{3}$ and again ignore the third order constant terms yields

$$
F_{X}^{3} \approx \frac{3 K \Delta_{3}^{R}\left(\Delta_{1}^{S}\right)^{2}}{2(K-1)^{2}} \cos \{3 \Omega \tau\}
$$

Next use (10), (A.2), $F_{X}^{1}, F_{Y}^{1}, F_{X}^{2}, F_{Y}^{2}$ and the above approximation of $F_{X}^{3}$ to find the simplified linear system

$$
\begin{aligned}
X^{\prime \prime}+2 \zeta X^{\prime}+\widehat{K} X= & H \Omega^{2} \cos \left\{\Omega \tau+\alpha_{h}\right\}-\frac{\Delta_{1}^{S}}{K} \\
& +\frac{3 K \Delta_{3}^{R}\left(\Delta_{1}^{S}\right)^{2}}{2(K-1)^{2}} \cos \{3 \Omega \tau\}, \\
Y^{\prime \prime}+2 \zeta Y^{\prime}+\widehat{K} Y= & H \Omega^{2} \sin \left\{\Omega \tau+\alpha_{h}\right\} \\
& +\frac{3 K \Delta_{3}^{R}\left(\Delta_{1}^{S}\right)^{2}}{2(K-1)^{2}} \sin \{3 \Omega \tau\}
\end{aligned}
$$

Equation (A.9) has a steady state solution of the form

$$
\begin{aligned}
& X(t)=R_{H} \cos \left\{\Omega \tau+\alpha_{h}-v_{H}\right\}+R_{\Delta} \cos \left\{3 \Omega \tau-v_{\Delta}\right\}, \\
& Y(t)=R_{H} \sin \left\{\Omega \tau+\alpha_{h}-v_{H}\right\}+R_{\Delta} \sin \left\{3 \Omega \tau-v_{\Delta}\right\},
\end{aligned}
$$

with $X_{E}, Y_{E}, R_{H}$ and $v_{H}$ as in (A.5). $R_{\Delta}$ and $v_{\Delta}$ yield

$$
\begin{aligned}
& R_{\Delta}=\frac{3 K \Delta_{3}^{R}\left(\Delta_{1}^{S}\right)^{2}}{2(K-1)^{2} \sqrt{\left(\widehat{K}-4 \Omega^{2}\right)^{2}+16 \Omega^{2} \zeta^{2}}}, \\
& v_{\Delta}=\arctan \left\{\frac{4 \zeta \Omega}{\widehat{K}-4 \Omega^{2}}\right\} .
\end{aligned}
$$

To find a condition for whirling zeroes we again ask for the existence of solutions to the equation $Y^{\prime} \widehat{X}-\widehat{X}^{\prime} Y=0$, where $\widehat{X}=X-X_{E}$. Using (A.10) we obtain

$$
\frac{R_{H}^{2}+2 R_{\Delta}^{2}}{3 R_{H} R_{\Delta}}=-\cos \left\{2 \Omega \tau-\alpha_{h}+v_{H}-v_{\Delta}\right\} .
$$

Equation (A.12) has either no solution, two solutions (if left hand side is 1) or four solutions symmetrically located in the $X Y$-plane, as expected from the simulations in Figure 3. The condition for existence is

$$
R_{H}^{2}+2 R_{\Delta}^{2} \leq 3 R_{H} R_{\Delta}
$$

and we proceed as in the former case to arrive at the desired inequality.

\section{A.3 Stator triangularity with rotor ovality}

First we note that $F_{X}^{1}=X / K$ and $F_{Y}^{1}=Y / K$. The second order terms yield

$$
\begin{aligned}
& F_{X}^{2}=\frac{3}{2 K} \Delta_{2}^{R}\left(Y \sin \{2 \Omega \tau\}+\left(X-\Delta_{3}^{S}\right) \cos \{2 \Omega \tau\}\right), \\
& F_{Y}^{2}=\frac{3}{2 K} \Delta_{2}^{R}\left(\left(X+\Delta_{3}^{S}\right) \sin \{2 \Omega \tau\}+Y \cos \{2 \Omega \tau\}\right) .
\end{aligned}
$$

As in the former cases we assume that the rotor moves close to an equilibrium. In this case, due to symmetry, this equilibrium is the origin. The simplifying system becomes

$$
\begin{aligned}
X^{\prime \prime}+2 \zeta X^{\prime}+\widehat{K} X= & H \Omega^{2} \cos \left\{\Omega \tau+\alpha_{h}\right\} \\
& -\frac{3 \Delta_{2}^{R} \Delta_{3}^{S}}{2 K} \cos \{2 \Omega \tau\}, \\
Y^{\prime \prime}+2 \zeta Y^{\prime}+\widehat{K} Y= & H \Omega^{2} \sin \left\{\Omega \tau+\alpha_{h}\right\} \\
& +\frac{3 \Delta_{2}^{R} \Delta_{3}^{S}}{2 K} \sin \{2 \Omega \tau\} .
\end{aligned}
$$

Equation (A.14) has a steady state solution of the form (A.4) with $X_{E}=Y_{E}=0, R_{H}, v_{H}, v_{\Delta}$ as in (A.5) and 


$$
R_{\Delta}=R_{\Delta}^{X}=-R_{\Delta}^{Y}=\frac{3 \Delta_{2}^{R} \Delta_{3}^{S}}{2 K \sqrt{\left(\widehat{K}-4 \Omega^{2}\right)^{2}+16 \Omega^{2} \zeta^{2}}} .
$$

Proceeding as in the former cases we find the following condition for whirling zeroes,

$$
\frac{R_{H}^{2}-2 R_{\Delta}^{2}}{R_{H} R_{\Delta}}=\cos \left\{3 \Omega \tau+\alpha_{h}-\left(v_{H}+v_{\Delta}\right)\right\}
$$

Equation (A.16) has either no solution, three solutions (if left hand side is 1) or six solutions symmetrically located in the $X Y$-plane, as expected from the simulations. In this case the condition for existence is

$$
\left|R_{H}^{2}-2 R_{\Delta}^{2}\right| \leq R_{H} R_{\Delta}
$$

and we proceed as in the former cases to arrive at the desired inequality.

\section{A.4 Stator ovality with rotor triangularity}

Similar to the former cases $F_{X}^{1}=X / K$ and $F_{Y}^{1}=Y / K$. The second order terms are given by

$$
\begin{aligned}
& F_{X}^{2}=-\frac{3}{2 K} \Delta_{2}^{S}\left(\Delta_{3}^{R} \cos \{3 \Omega \tau\}+X\right), \\
& F_{Y}^{2}=-\frac{3}{2 K} \Delta_{2}^{S}\left(\Delta_{3}^{R} \sin \{3 \Omega \tau\}-Y\right) .
\end{aligned}
$$

Note that in this case the second order terms $F_{X}^{2}$ and $F_{Y}^{2}$ are simpler in the sense of the dependence of $X$ and $Y$, and we do not need to simplify further by assuming the rotor center close to an equilibrium. The simplified linear system yields

$$
\begin{aligned}
X^{\prime \prime}+2 \zeta X^{\prime}+K_{X} X= & H \Omega^{2} \cos \left\{\Omega \tau+\alpha_{h}\right\} \\
& -\frac{3 \Delta_{2}^{S} \Delta_{3}^{R}}{2 K} \cos \{3 \Omega \tau\} \\
Y^{\prime \prime}+2 \zeta Y^{\prime}+K_{Y} Y= & H \Omega^{2} \sin \left\{\Omega \tau+\alpha_{h}\right\} \\
& -\frac{3 \Delta_{2}^{S} \Delta_{3}^{R}}{2 K} \sin \{3 \Omega \tau\}
\end{aligned}
$$

where $K_{X}=1-K^{-1}+3 \Delta_{2}^{S} / 2$ and $K_{Y}=1-K^{-1}-3 \Delta_{2}^{S} / 2$. Equation (A.17) has a steady state solution of the form

$$
\begin{aligned}
& X(t)=R_{H}^{X} \cos \left\{\Omega \tau+\alpha_{h}-v_{H}^{X}\right\}+R_{\Delta}^{X} \cos \left\{3 \Omega \tau-v_{\Delta}^{X}\right\}, \\
& Y(t)=R_{H}^{Y} \sin \left\{\Omega \tau+\alpha_{h}-v_{H}^{Y}\right\}+R_{\Delta}^{Y} \sin \left\{3 \Omega \tau-v_{\Delta}^{Y}\right\},
\end{aligned}
$$

where

see equation (A.19) at the bottom of this page.

Due to the ovality of the stator the amplitude in $X$ will be smaller than the amplitude in $Y$, which can be seen in the formulas by the different stiffness $K_{X}$ and $K_{Y}$. Due to the difference in the amplitudes the condition for a whirling zero becomes more complicated in this case. However, simplifying by ignoring this difference yields a similar situation as in the former cases, and still an informative analytic expression. Hence, we replace $K_{X}$ and $K_{Y}$ in (A.19) by $\widehat{K}=1-K^{-1}$ and then we obtain $R_{H}^{X}=R_{H}^{Y}:=R_{H}$, $v_{H}^{X}=v_{H}^{Y}:=v_{H}, R_{\Delta}^{X}=R_{\Delta}^{Y}:=R_{\Delta}$ and $v_{\Delta}^{X}=v_{\Delta}^{Y}:=v_{\Delta}$. Now proceeding as in the former cases we find that the condition for whirling zeroes is

$$
\frac{R_{H}^{2}+2 R_{\Delta}^{2}}{3 R_{H} R_{\Delta}}=\cos \left\{2 \Omega \tau-\alpha_{h}+v_{H}-v_{\Delta}\right\}
$$

Equation (A.20) has either no solution, two solutions or four solutions symmetrically located in the $X Y$-plane. The condition for existence becomes as in the first case, that is

$$
R_{H}^{2}+2 R_{\Delta}^{2} \leq 3 R_{H} R_{\Delta}
$$

and we proceed as in the former cases to arrive at the desired inequality. The proof of Theorem 1 is complete.

\section{A.5 Verification of the simplified equations of motion}

Figure 10 shows comparisons between some solutions of the simplified linear equations of motions (A.3), (A.9), (A.14), (A.17), derived above, and the original equation of motion (10). The agrement can be seen to be rather good.

$$
\begin{aligned}
& R_{H}^{X}=\frac{H \Omega^{2}}{\sqrt{\left(K_{X}-\Omega^{2}\right)^{2}+4 \zeta^{2} \Omega^{2}}}, v_{H}^{X}=\arctan \left\{\frac{2 \zeta \Omega}{K_{X}-\Omega^{2}}\right\} \\
& R_{H}^{Y}=\frac{H \Omega^{2}}{\sqrt{\left(K_{Y}-\Omega^{2}\right)^{2}+4 \zeta^{2} \Omega^{2}}}, v_{H}^{Y}=\arctan \left\{\frac{2 \zeta \Omega}{K_{Y}-\Omega^{2}}\right\} \\
& R_{\Delta}^{X}=\frac{3 \Delta_{2}^{R} \Delta_{3}^{S}}{2 K \sqrt{\left(K_{X}-4 \Omega^{2}\right)^{2}+16 \Omega^{2} \zeta^{2}}}, v_{\Delta}^{X}=\arctan \left\{\frac{4 \zeta \Omega}{K_{X}-4 \Omega^{2}}\right\}+\pi \\
& R_{\Delta}^{Y}=\frac{3 \Delta_{2}^{R} \Delta_{3}^{S}}{2 K \sqrt{\left(K_{Y}-4 \Omega^{2}\right)^{2}+16 \Omega^{2} \zeta^{2}}}, v_{\Delta}^{Y}=\arctan \left\{\frac{4 \zeta \Omega}{K_{Y}-4 \Omega^{2}}\right\}+\pi
\end{aligned}
$$


N.L.P. Lundström et al.: Small shape deviations causes complex dynamics in large electric generators
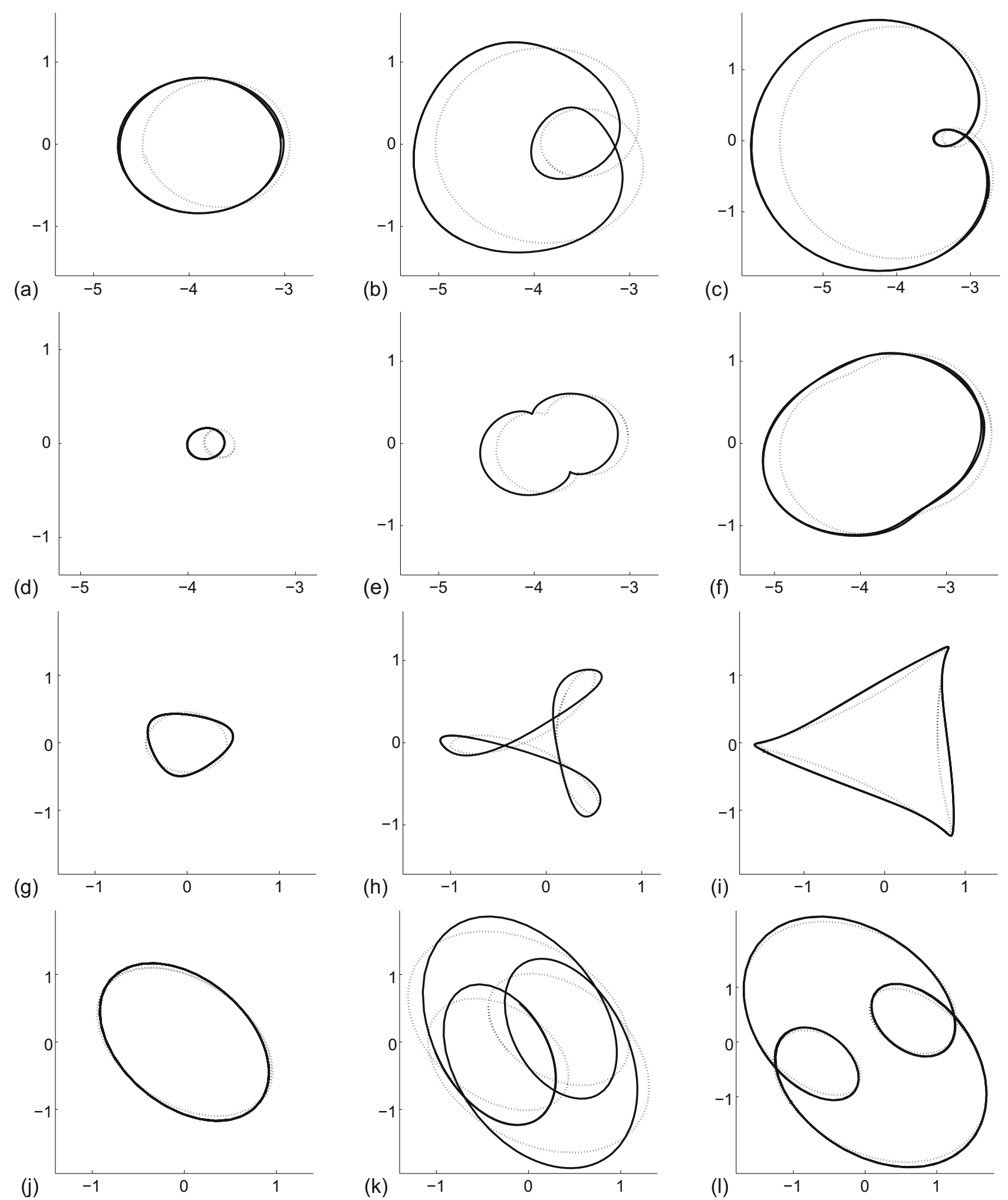

Fig. 10. Comparisons between the simplified linear equations of motions (dotted) and the original equation of motion (10) (solid). All shape deviations are 5\%. $H$ is increasing from left to right; $H=0, H=0.015, H=0.1$. (a)-(c): stator eccentricity with rotor ovality, (d)-(f): stator eccentricity with rotor triangularity, (g)-(i): stator triangularity with rotor ovality, (j)-(l): stator ovality with rotor triangularity. Horizontal axis shows $100 X$ and vertical axis shows $100 Y$. 